Das Erbe der Apartheid als Hindernis für nachhaltigen Nahverkehr in Südafrika

\section{Renaissance des Fahrrades?}

\section{Auch nach dem Ende der politischen Apartheid bestehen in Südafrika die Raum- strukfuren der Apartheid in den Ballungsräumen weiter. Die resultierenden Mobili- tätsprobleme der ärmeren, mehrheitlich schwarzen Bevölkerung sind so groß, dass sie die gesamtgesellschaftliche Überwindung der Apartheid verhindern. Ein in allen Dimensionen nachhaltiger Beitrag liegt in der Wiederbelebung der Fahrrad- kultur.}

$\mathrm{E}$ Von Christof Hertel rgebnis der Apartheidpolitik ist eine ausgeprägte „race-class dichotomy“ (1). Die sozioökonomische Spaltung der südafrikanischen Gesellschaft durch eine Vielzahl von Apartheidgesetzen, die sich insbesondere gegen schwarze Bevölkerungsgruppen richteten und diese in allen Lebensbereichen benachteiligten, fand ihren räumlichen Niederschlag in der „Apartheid-City" (2):

- Getrennte Wohngebiete für verschiedene Rassen: Die schwarzen Bevölkerungsgruppen wurden in sogenannte Townships am Rande der Stadt und ab 1960 auch weiter entfernt in die sogenannten Homelands zwangsumgesiedelt.

- Monofunktionale, eintönige Townships: Die Struktur der Townships ist geprägt von monotonen, kleinen Einzelhäusern. Infrastrukturen, wie asphaltierte Fußwege und Straßen, weiterführende Schulen, Supermärkte, Einkaufszentren, Dienstleister und Banken sind kaum vorhanden. Die öffentliche Verkehrsanbindung der Townships ist auf die Arbeitsplatzzentren und -zeiten in den Industriegebieten und Bergwerksstandorten ausgerichtet und besteht häufig aus einer Eisenbahnlinie am Rande der Townships sowie unvertakteten Busverkehren.

- Starke Abschottung: Die Townships wurden durch Pufferzonen von den Wohngebieten der weißen Bevölkerung strategisch abgeschottet. Dabei wurden Bergrücken, Bäche und Flüsse als natürliche Barrieren und Schnellstraßen, Eisenbahndämme, Kanäle, Müllhalden und Industriezonen als menschgemachte, schwer überwindbare Barrieren planerisch genutzt. Entstanden sind autonome, unzusammenhängende Stadtteil"Zellen".

Diese in Jahrzehnten der Apartheid gewachsenen räumlich-sozialen Strukturen können nicht mit einer politischen Verfassungsänderung aufgehoben werden. Vielmehr sind sie auch heute noch - unterstützt durch die ökonomischen Mecha- nismen wie Bodenpreise, Arbeitsmarkt, Mobilitätskosten und Standortwahl - feststellbar und vertiefen sich zum Teil weiter.

\section{- Mobilitätsprobleme als Folge}

Die Apartheidpolitik hat nicht nur raumstrukturelle, sichtbare Barrieren bewirkt, sondern sie hat auch das Mobilitätspotenzial der Bewohner der Townships beeinflusst und verringert die Mobilitätschancen der Township-Bewohner noch heute.

Die Lage und das Fehlen von leistungsfähiger und kostengünstiger Infrastruktur in den Townships führt zu weiten Wegen zu Arbeitsplätzen, Einkaufsmöglichkeiten und Innenstädten. Beispielsweise liegen die im Rahmen einer Diplomarbeit untersuchten Townships Atteridgeville, Ivory Park und Winterveld zwischen 10 und 45 Kilometer von Pretoria entfernt (3). Bis zum nächstgelegenen Bahnhof müssen von manchen Bereichen der Townships bis zu sechs bzw. zehn Kilometer zurückgelegt werden.

Die Pufferzonen führen für die Bewohner der Townships zu langen Umwegen und Gefährdungen. Oft können sie Wege nicht ohne Verkehrsmittel zurücklegen oder bestimmte Ziele nicht erreichen, $\mathrm{da}$ die Townships nur über eine geringe Anzahl an definierten Einfahrten verlassen werden können und die Überquerung der Barrieren gefährlich ist.

Wichtigster Beeinflussungspunkt der individuellen Mobilität ist das Einkommen, welches massiv durch die Apartheidpolitik gespreizt wurde. Nicht zuletzt als Folge des geringen Einkommens ist der Grad der Verkehrsmittelverfügbarkeit in den Townships gering. In Atteridgeville besitzt durchschnittlich nur ein Haushalt von zehn ein Auto, während in anderen ,weißen“ Stadtteilen Haushalte durchschnittlich über zwei Autos verfügen (4).

Aber auch die ineffiziente Organisation des öffentlichen Nahverkehrs durch noch immer separat organisierte Busgesellschaften für (ehemals) die schwarze und für die weiße Bevölkerungsgruppe, das Fehlen von Verbundfahrscheinen für Bahn, Bus und privaten Minibussen und der Mangel an adä- quaten Verkehrswegen (5) verringert die Mobilitätspotenziale der Township-Bewohner und führt so zu einer „Race-Mobility Dichotomy“.

Das früher häufig genutzte Fahrrad verschwand in den letzten fünfzig Jahren fast völlig aus dem Straßenbild. Der Modal-Split Anteil des Fahrrades liegt in Pretoria bei sehr geringen 0,8 Prozent. Der Anteil bei Schulwegen in Atteridgeville ist mit 1,4 Prozent nur unwesentlich höher (6).

\section{- Nachhaltiges Verkehrsmittel Fahrrad}

Unter den gegebenen Bedingungen - weite Wege, geringes Einkommen - könnte eine Strategie zur Verbesserung der Mobilität der Township-Bewohner in der Wiederbelebung der Fahrradkultur liegen. Mit dem Fahrrad steht ein kostengünstiges, robustes, umweltschonendes und damit sowohl ökologisch als auch ökonomisch hocheffizientes Verkehrsmittel zur Verfügung. In Interviews mit in den Townships angetroffenen erwachsenen Fahrradfahrern zeigten sich vor allem folgende Nutzungsmuster:

- Für den Arbeitsweg wird das Fahrrad genutzt, wenn mit öffentlichen Verkehrsmitteln viele Umstiege und damit hohe Fahrtkosten verbunden sind, oder wenn der Arbeitsort mit dem Fahrrad gut erreichbar ist.

- Das Fahrrad wird auch für eine Vielzahl an $B e$ sorgungen genutzt: für den Einkaufsweg, um Wasser zu transportieren, oder um Kinder in den Kindergarten zu bringen.

- In der Freizeit dient das Fahrrad als Fortbewegungsmittel, zum Beispiel für Verwandten- und Gottesdienstbesuche, oder als Trainingsgerät für einige Radsportenthusiasten.

- Für Unternehmer ist das Fahrrad sowohl Transportmittel für den Einkauf in entfernteren Großmärkten, als auch ein Arbeitsgerät, von dem direkt der Verkauf von Waren innerhalb des Townships vorgenommen wird.

\section{Nutzungshindernisse}

Trotz dieser vielfältigen Einsatzmöglichkeiten ist der Anteil des Fahrrades am Modal-Split der Townshipbewohner bisher sehr gering. Auch hierfür lieferte die Untersuchung einige Erklärungsansätze:

- Sicherheit: Verkehrssicherheit - im Jahr 1995 waren 40 Prozent der Verkehrstoten Fußgänger und Kriminalitätsfurcht sind für nichtmotorisierte Verkehrsteilnehmer Gründe, Wege nicht oder im Schutze eines öffentlichen Verkehrsmittels zurückzulegen. Aus Furcht vor Diebstahl werden Fahrräder nicht am Bahnhof abgestellt und somit kaum intermodale Wegeketten zurückgelegt. 
- Infrastruktur: In den Townships gibt es bisher fast keine Fahrradläden, Ersatzteilverkäufer oder Radwerkstätten. Beispielsweise gibt es in Atteridgeville für rund 150.000 Einwohner nur ein Hinterhoffahrradgeschäft, die nächste Ersatzteilversorgung liegt in Ivory Park sieben bis 10 Kilometer und in Winterveld 30 bis 35 Kilometer entfernt. Daher werden viele Räder auf Verschleiß gefahren. Kaputte Fahrräder dienen als Ersatzteillieferant für Räder von Freunden.

- Finanzierung: In der Regel verfügen die Township-Bewohner über keine finanziellen Reserven, sodass viele die Anschaffung eines Fahrrades bzw. die Reparaturen und Ersatzteile nicht bezahlen können. Dabei könnten sich diese Ausgaben innerhalb weniger Wochen und Monate amortisieren, wenn beispielsweise die teuren Fahrten mit dem Minibus wegfallen.

- Kommunikationsdefizite: Viele Bewohner, die bisher nicht selbst Fahrrad fahren, wissen in der Regel nicht um die Einsatzmöglichkeiten des Fahrrades bzw. ihnen fehlt das Know-how bezüglich des Fahrens und der Wartung. Zudem hat auch unter ihnen das Auto ein besseres Image als das Fahrrad.

\section{- Überwindung mit Hilfe von Afribike}

Diese Hindernisse aus dem Weg zu räumen und die Fahrradkultur wiederzubeleben, hat sich die Nichtregierungsorganisation Afribike zum Ziel gesetzt (7). In Eigeninitiative hat sie verschiedene Projekte angeschoben. Inzwischen hat Afribike das ,Shova Kalula National Bicycle Demonstration Programme“ initiiert und wird vom Verkehrsministerium gefördert. Folgende Maßnahmen sollen im Rahmen von Pilotprojekten umgesetzt werden:

- In Atteridgeville sowie auf dem Weg in die Innenstadt Pretorias sollen Fahrradwege geplant und bewachte Fahrradparkplätze an den Bahnhöfen entstehen.

- In Ivory-Park wurde bereits ein Fahrradladen mit Werkstatt eingerichtet, der durch lokale Arbeitskräfte geleitet wird, die von Afribike ausgebildet wurden. Dieser Fahrradladen verkauft neue und aufgearbeitete Alltagsräder sowie Ersatzteile.

- Für bedürftige Schüler werden Work \& BikeProgramme angeboten, in denen sie ein Secondhand-Fahrrad unter Anleitung aufarbeiten, dabei die Wartung erlernen und im Fahrradladen mithelfen. Nach Absolvierung des Kurses können sie das Fahrrad behalten oder subventioniert kaufen.

- Landesweit wird durch Öffentlichkeitsarbeit und Lobbying die Aufmerksamkeit auf das Nahver- kehrsmittel Fahrrad und seine Vorteile gelenkt und diese auch bei den Projekten vor Ort mit gezielten Image- und Informationskampagnen unterstïtzt.

- Langfristig ist die Umsetzung eines eigenständigen Fahrradtyps AfriBike vorgesehen, welches die Kriterien kostengünstig, robust, modern und attraktiv in einem Fahrrad bündelt.

Afribike setzt damit an den zentralen Problempunkten Verkehrssicherheit, allgemeine Sicherheit, Infrastruktur, Finanzierung und Information an. Daher kann mit Hilfe des Fahrrades die Situation der ärmeren Bevölkerung in den Townships auf eine sehr direkte Art verbessert werden. Die Raumstrukturen der Apartheid werden zwar nicht beseitigt, jedoch überwunden. Durch diese Überwindung kann langfristig auch die Infrastruktur verbessert werden, wenn nun beispielsweise mehr Verkäufer mit Fahrrädern in die Townships kommen. Arbeitsplätze können geschaffen werden.

Das Fahrrad ist dabei sicher nicht für jeden und für jede Situation geeignet, doch sein Einsatzpotenzial ist wesentlich größer, wenn fahrradfreundlichere Strukturen geschaffen werden.

\section{Anmerkungen}

(1) Jürgens, U.: Gemischtrassige Wohngebiete in südafrikanischen Städten. Kiel 1991.

(2) Davies, R.J.: The Spatial Formation of the South African City. In: GeoJournal, 1981, Suppl. (2), S. 59-72.

(3) Hertel, C.: Apartheidbedingte Mobilitätsprobleme und Strategien zu ihrer Überwindung in südafrikanischen Townships. Freie Universitüt Berlin 2001, unveröffentlichte Diplomarbeit.

(4) GPMC Greater Pretoria Metropolitan Council: Household Travel Survey 1998/99- Final Report. Pretoria 2000.

(5) National Department of Transport: Moving South Africa. The Action Agenda. Pretoria 1999.

(6) City Council of Pretoria: Bicycle Study for the Pretoria Municipal Area: Status Quo Investigation (Final Draft). Pretoria 1999.

(7) Siehe www.afribike.org

\section{Der Autor}

Christof Hertel ist wissenschaftlicher Mitarbeiter an der Technischen Universität Hamburg-Harburg. Kontakt: TU HH, Arbeitsbereich 1-10 Verkehrssysteme und Logistik, 21071 Hamburg. Tel. 040/ 42878-3903, Fax -2728, E-Mail: c.hertel@tu-harburg.de politische ökologie 79

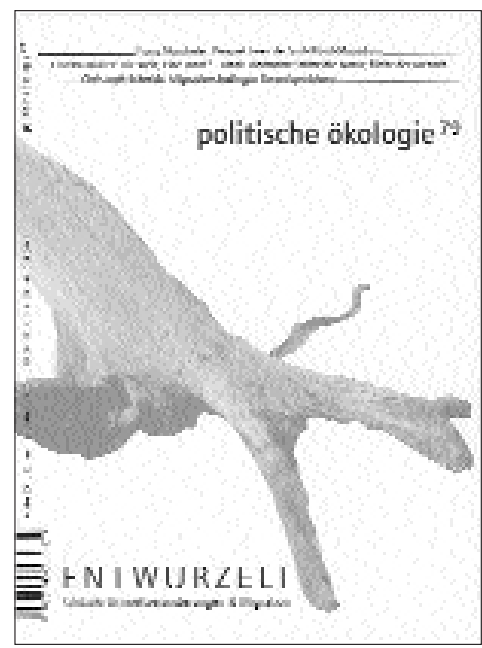

\section{Entwurzelt}

\section{Globale Umweltveränderung \& Migration}

Land unter für Tuvalu! Dem polynesischen Inselstaat droht aufgrund des steigenden Meeresspiegels die Überflutung. Wer trägt hierfür die Verantwortung und wohin mit den Insulanern?

Die Zerstörung der natürlichen Lebensgrundlagen ist neben politischen und wirtschaftlichen Gründen die Hauptursache für eine ansteigende Migrationswelle. Vor dieser fürchten sich die reichen Industrieländer, die als deren Hauptverursacher gelten. Diese müssen nun einen Weg finden, sich mit "dem globalen Marsch" zu arrangieren.

Die politische ökologie 79 widmet sich diesmal dem Themenkomplex Migration, Umwelt und Integration.

Mit Beiträgen von J. Oltmer, C. Müller, F. Nuscheler, F. Biermann, G. Schön, u.v.m.

\section{www.oekom.de}

erhältlich bei pan adress

Semmelweisstraße 8, D-82152 Planegg

Fon $++49 /(0) 89 / 857$ 09-155

Fax $++49 /(0) 89 / 857$ 09-131

E-Mail kontakt@oekom.de 
(c) 20I0 Authors; licensee IÖW and oekom verlag. This is an article distributed under the terms of the Creative Commons Attribution Non-Commercial No Derivates License (http://creativecommons.org/licenses/by-nc-nd/3.o/), which permits unrestricted use, distribution, and reproduction in any medium, provided the original work is properly cited. 